\title{
Comparison of Two Multilevel Architectures for Envelope Amplifier
}

\author{
M. Vasić, O. Garcia, J.A. Oliver, P. Alou, D. Diaz, J.A. Cobos \\ Universidad Politécnica de Madrid, Centro de Electrónica Industrial \\ José Gutierrez Abacal 2, 28006 Madrid \\ miroslav.vasic@upm.es
}

\begin{abstract}
Modern transmitters usually have to amplify and transmit signals with simultaneous envelope and phase modulation. Due to this property of the transmitted signal, linear power amplifiers (class $\mathrm{A}, \mathrm{B}$ or $\mathbf{A B}$ ) are usually used as a solution for the power amplifier stage. These amplifiers have high linearity, but suffer from low efficiency when the transmitted signal has low peak-to-average power ratio. The Kahn envelope elimination and restoration (EER) technique is used to enhance efficiency of RF transmitters, by combining highly efficient, nonlinear RF amplifier (class D or E) with a highly efficient envelope amplifier in order to obtain linear and highly efficient RF amplifier. This paper compares two solutions for the envelope amplifier based on a combination of multilevel converter and linear regulator. The solutions are compared regarding their efficiency, size and weight. Both solutions can reproduce any signal with maximal spectral component of $1 \mathrm{MHz}$ and give instantaneous maximal power of 50 W. The efficiency measurements show that when the signals with low average value are transmitted, the implemented prototypes have up to $19 \%$ higher efficiency than linear regulator that is used as a conventional solution.
\end{abstract}

\section{INTRODUCTION}

In the modern world of today, the demand for broadband and wireless services is growing on a daily basis. One of direct consequences of this growth is certainly the growth of the networks that have to provide these services and the problem is their energy consumption. Some estimations showed that a $1 \%$ of planet's global energy consumption in 2007 was made by telecommunication industry [1]. In [2] is explained that the efficiency of the first generation $3 \mathrm{G}$ radio base stations is just few percents, and that the efficiency of the employed power amplifiers is just $6 \%$. The impact of power amplifier's efficiency can be seen in the information that if the power amplifiers could improve its efficiency by $10 \%$ the overall efficiency would be raised by $6 \%$. Therefore, the questions are rising. Why do the power amplifiers have low efficiency? Is there a possibility to increase it?

The transmitters usually employ digital modulations such as QPSK combined with spread spectrum techniques like CDMA or WCDMA. The modulated signals are later amplified by using highly linear, but low efficient linear amplifiers like class A or class B amplifiers. In the ideal case, when a sine wave is amplified, the maximal efficiency for class A and class B amplifiers reaches $50 \%$ and $78.5 \%$ respectively. However, the theoretical value is usually reduced by the factor of 0.8 to 0.85 due to various losses [3]. Additionally, the maximal efficiency is calculated in the case of the signal that has constant amplitude. The signals that are amplified by the PA and latter transmitted usually have time varying envelope, so that the only way to calculate the efficiency of the PA is to use the probability density function [4]. The probability density function of the envelope gives the relative amount of time a signal spends at various amplitudes, Fig. 1 [4]. Frequency modulated signals or constant wave signals have constant envelope and, therefore, linear power amplifiers or class $\mathrm{E}$ amplifiers could be optimal solution for the transmitter's PA. On the other hand, noise and multiple carriers have Rayleighdistributed envelope and high peak-to-average power ratio (PAPR) [3].

Instantaneous efficiency is the efficiency of the PA at one certain output level. The instantaneous efficiency of class A and class B amplifier in the case of various sine wave amplitudes is shown in Fig. 2. From Fig. 1 and Fig. 2 it can be seen that the signals with high PAPR have the highest probability in the zone where linear amplifiers have very low efficiency (approximately 15\% for class B) and that is the main reason for low efficiency of these PA applied in RF systems. In [5] is explained that class A and class B amplifiers have efficiency of $5 \%$ and $28 \%$ respectively if a signal with a Rayleigh's distribution is transmitted.

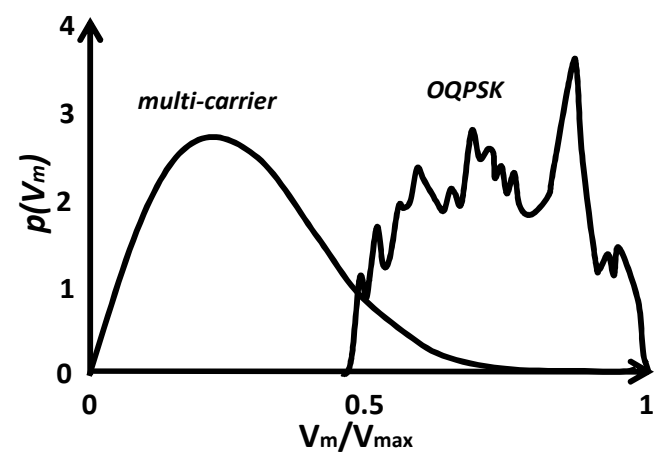

Fig. 1. Probability density function of the signal's envelope for different

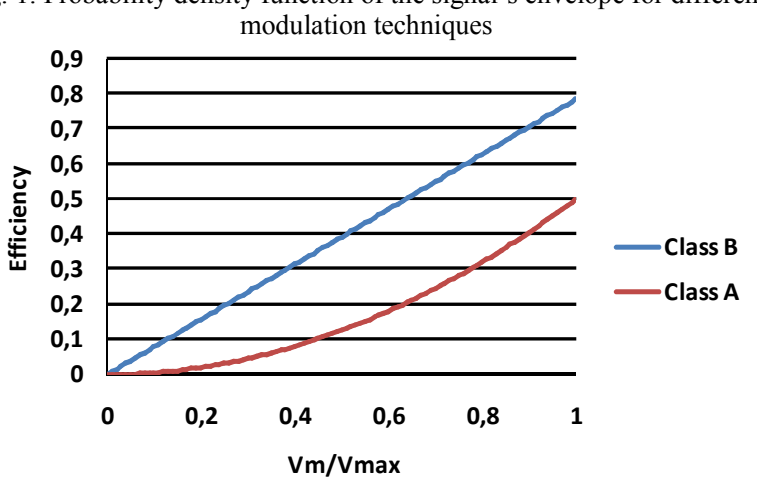

Fig. 2. Instantaneous efficiency of class A and class B amplifier in the case of various sine wave amplitudes 
The Kahn envelope elimination and restoration (EER) technique is used to enhance efficiency of RF transmitter. Fig. 3 shows block diagram of one EER transmitter. This technique combines a highly efficient, but nonlinear RF PA (class D or class E for example) with a highly efficient envelope amplifier to implement high-efficiency linear RF PA [6].

The basis for EER is the equivalence of any narrowband signal to simultaneous amplitude (envelope) and phase modulation:

$$
\begin{array}{r}
V_{R F}(t)=I(t) \cos (2 \pi f t)-Q(t) \sin (2 \pi f t)= \\
=A(t) \cos (2 \pi f t+\theta(t)) \\
\theta(\mathrm{t})=\operatorname{arctg}\left(\frac{Q(t)}{I(t)}\right) \quad A(t)=\sqrt{I(t)^{2}+Q(t)^{2}}
\end{array}
$$

where $f$ is the carrier frequency, $Q(t)$ and $I(t)$ are modulated signals.

Therefore, analyzing the equation (1), it can be concluded that the nonlinear RF PA should be used to change the phase of transmitted signal, and the envelope amplifier to apply amplitude modulation. By applying this technique it is possible to achieve 3 to 5 times better efficiency comparing with standard class A and class B amplifiers [3, 4, 7, 8].

The envelope amplifier should have fast dynamic response, high efficiency and minimal interference with the output spectrum of the transmitter. In the state of the art, several solutions for the envelope amplifier can be found, such as a simple buck converter (class $\mathrm{S}$ modulator) in $[9,10]$, multiphase buck converter in [11], three-level converter in [12] or linear assisted switching amplifier [13, 14]. These solutions do not exceed the bandwidth of few hundred $\mathrm{kHz}$ and the output power is from the range of $\mathrm{mW}$ up to several tens of watts [4]. In [15, 16] buck converter is integrated and the switching frequency is in range of several $\mathrm{MHz}$, but with small output power, in the range of $\mathrm{mW}$.

The envelope amplifier needs to have high efficiency, and therefore, a dc-dc converter would be a first idea for the solution. Nevertheless, in order to provide high bandwidth that is necessary to follow the envelope reference, these converters have to use switching frequency up to five times higher than the requested bandwidth (for the bandwidth of $1 \mathrm{MHz}$ it would be necessary to apply switching frequency of, at least, $5 \mathrm{MHz}$ ) [17]. The efficiency of dc-dc converters drops heavily when the switching frequency is increased; therefore, the efficiency of whole system drops as well. The second problem is the output filter of the converter, because its design can be very complicated due to very strict restrictions regarding the voltage ripple and spectral interference. In some papers, it is proposed to use double LC filter [9], but the use of this filter could decrease the maximum bandwidth.

The solution based on a multilevel converter in series with a linear regulator is presented in [18]. It is shown that this solution can reproduce $2 \mathrm{MHz}$ sine wave, with low spectral distortion and provides $50 \mathrm{~W}$ of instantaneous power. This topology operates at relatively low switching frequency and without additional output filter because the linear regulator filters all the noise and ripple that comes from the multilevel convert.

In this paper two different implementations of this topology are compared regarding its efficiency, complexity, size and possibility of integration.

\section{ARCHITECTURE OF THE ENVELOPE AMPLIFIER}

The topology that is used for the envelope amplifier consists of a multilevel converter in series with a high slew rate linear regulator. The main idea of the solution can be seen in Fig. 4. The multilevel converter has to supply the linear regulator and it has to provide discrete voltage levels that are as close as possible to the output voltage of the envelope amplifier. If this is fulfilled, the power losses on the linear regulator will be minimal, because they are directly proportional to the difference of its input and output voltage. However, in order to guarantee correct work of the linear regulator, the output voltage of the multilevel converter always has to be higher than the output voltage of the linear regulator. Similar solution, but for lower frequencies and higher power is presented in [19]

The linear regulator can be designed to have very high bandwidth, and it should filter all the noise that could come from the multilevel converter. Therefore, the multilevel converter does not need any filter at its output and the design of the complicated filter as in the case of switched converters is avoided.

There are several possibilities to implement the multilevel converter for this application. The first one, architecture one, is to provide all the voltages that are needed at its output, and then to use a switching network as an analog multiplexer to select each one when it is necessary, Fig. 5. In the case when the switches are realized with MOSFETs, it is necessary to put diodes in series in order to guarantee energy flow to the load, and to avoid possible short circuits through MOSFET parasite diode.

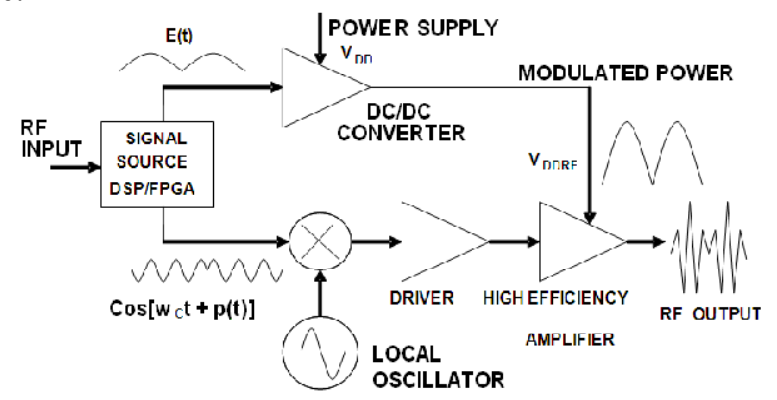

Fig. 3. Block Scheme of Kahn-technique Transmitter

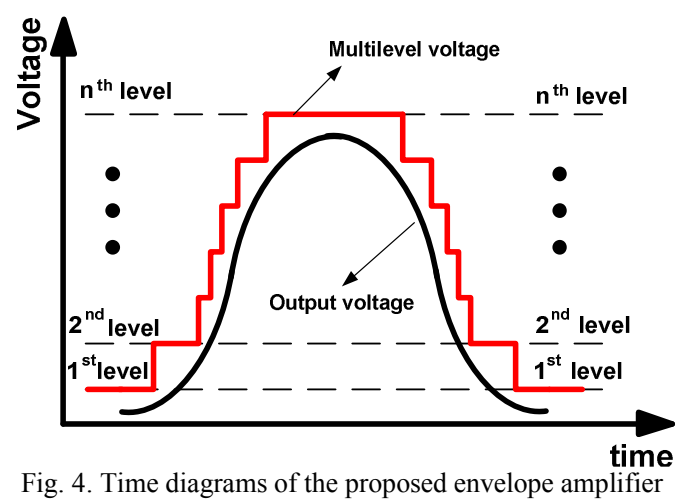

Fig. 4. Time diagrams of the proposed envelope amplifier 
The second solution is to use independent voltage cells that are put in series, and then to generate the output voltage as a combination of its voltages. These cells can be implemented to give just positive voltage (two-level cell, architecture two), or to produce positive and negative voltage (three-level cell, architecture three), Fig. 6. When the cell is turned on, it gives a constant voltage, and when it is turned off, it gives zero.

Therefore, the output of this converter can be represented as:

$$
v_{0}=\sum_{i=1}^{N} a_{i} V_{i}
$$

where, the $N$ is the total number of the implemented cells, $a_{i}$ takes the value of 0 when the $i^{\text {th }}$ cell is turned off and 1 or -1 when it is turned on and $V_{i}$ is the supply voltage of the $i^{\text {th }}$ cell.

Although there are multilevel solutions with only one input voltage, this one is different and it has to be supplied by several voltages. The reason lies in the fact that the reference signal does not have to be symmetric, like it is in the case when multilevel converters are used for inverter applications. The implemented design guarantees that each cell will have its supply voltage all the time, regardless on the reference signal sent to the envelope amplifier.

Due to the independent voltages that have to be produced, it is obvious that it is required to introduce a single-input multiple-outputs stage that will generate all the needed voltages. In the case of the first multilevel solution, the output voltages are the voltage levels that are needed in the system, and they are all referenced to the ground. When the multilevel converter is implemented with two-level and three-level cells, the output voltages should be isolated and referenced to the different grounds. The cell's input voltage does not need to be regulated accurately, because the fine regulation will be done by the linear regulator that is connected in series with the multilevel converter. Additionally, in the case of three-level cell, the cell's input source has to be bidirectional, because, depending on the state of the switches, the source will sink or source the current to the load.

In this paper solutions that employ architectures one and two are compared.

In order to provide fair comparison of two different implementations, both solutions have the same number of levels and the same voltage distribution. The voltage levels are selected in order to maximize overall efficiency and the optimization of the voltage levels is explained in [18].

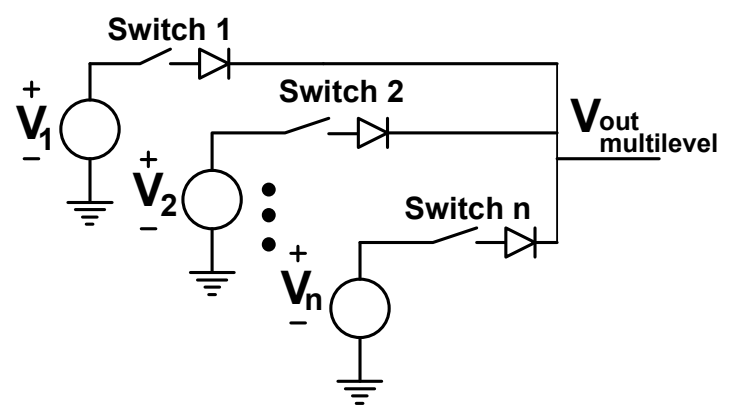

Fig. 5. Multilevel converter realized with independent supplies and analog multiplexer

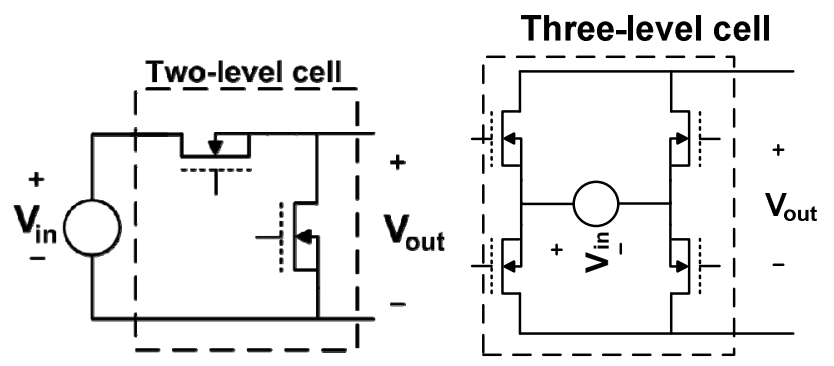

Fig. 6. Voltage cells that could be used as a solution to implement a multilevel converter

The envelope amplifiers that have been prototyped have following properties:

- The multilevel converter can reproduce three voltage levels

- The input voltage is $24 \mathrm{~V}$

- The output voltage can be $12 \mathrm{~V}, 18 \mathrm{~V}$ or $24 \mathrm{~V}$

The class E amplifier that is used for transmitter's phase modulation is supplied by the envelope amplifier and it behaves as a resistive load, approximately $12 \Omega$.

The advantage of this topology is that it provides high dynamics of the output voltage with increased efficiency comparing with linear regulator that is supplied with constant voltage and that its control is very simple and robust. The drawback is that each stage of the system (multiple-output converter, multilevel converter and linear regulator) needs to have very high efficiency, because the total efficiency is the product of individual efficiencies. However, it is still possible to achieve high overall efficiency, as it will be seen later.

\section{IMPLEMENTATION OF THE ARCHITECTURE ONE}

The multilevel converter for the architecture one is implemented using two converters based on switching capacitor in combination with an analog multiplexer. Both converters have the same topology and divide the input voltage [20], Fig. 7. The first converter is supplied by connecting its input terminals to the ground and $24 \mathrm{~V}$ voltage and its $12 \mathrm{~V}$ output voltage is referred to the ground. The second converter is supplied by connecting its input terminals between $12 \mathrm{~V}$ and $24 \mathrm{~V}$. Its output voltage is $6 \mathrm{~V}$, but this voltage is referred to the $12 \mathrm{~V}$ input, therefore, this output is, actually, $18 \mathrm{~V}$ output referring it to the ground, Fig. 8. The $24 \mathrm{~V}$ input voltage is directly provided to the analog multiplexer. One of the advantages of this solution is high efficiency that can provide converters based on switching capacitor and that it does not need any huge inductive component, and therefore it can be integrated easily. The disadvantage is that the switching noise or any noise that comes from the input voltage is poorly filtered and this could be a problem for the linear regulator depending on its bandwidth. In order to decrease the propagation of the switching noise to the output and to other system parts, small LC filters are introduced at the outputs of these two converters.

As it is shown in Fig. 5, the analog multiplexer consists of set of switches that are generally realized as a MOSFET in series with a diode. The diode is necessary in order to guarantee that independent voltage sources cannot be short-circuited through MOSFET's parasitic diode. However, in the case of $24 \mathrm{~V}$ 
voltage source only a MOSFET can be used, because there is not any higher voltage source in the system. Similar conclusion can be made in the case of $12 \mathrm{~V}$ source where only a diode can be used.

\section{IMPLEMENTATION OF THE ARCHITECTURE TWO}

Fig. 9 shows the block diagram of the implemented envelope amplifier based on architecture two. As it can be seen, a singleinput multiple-outputs converter is used to produce several independent voltages that are later combined by using two-level voltage cells.

In the case of the implemented solution in this paper, the single-input multiple-outputs converter is a flyback converter with three outputs. There are two $6 \mathrm{~V}$ outputs and one $12 \mathrm{~V}$ output. The minimum voltage of the multilevel converter is 12 $\mathrm{V}$ and, therefore, only the $6 \mathrm{~V}$ outputs are connected to twolevel cells.

\section{CONTROL OF ENVELOPE AMPLIFIER}

In both implementations of the envelope amplifier there can be recognized three stages. The first stage is a single-input multiple-output converter that has to provide independent voltages. The second stage applies summation or multiplexing of the independent voltages in order to produce voltage levels needed by the linear regulator. The last stage is the linear regulator that in its output reproduces the voltage needed by the power amplifier.

The first stage works in open loop when it is implemented with switching capacities. The switching frequency can be very low in order to maximize the efficiency of this stage. However, when a flyback converter is used, the first stage is controlled by a voltage feedback from one of flyback's outputs, because all the other outputs will follow the controlled one. The bandwidth of this stage does not have to be high; therefore, the switching frequency of the multiple-outputs flyback can be very low in order to increase its efficiency.

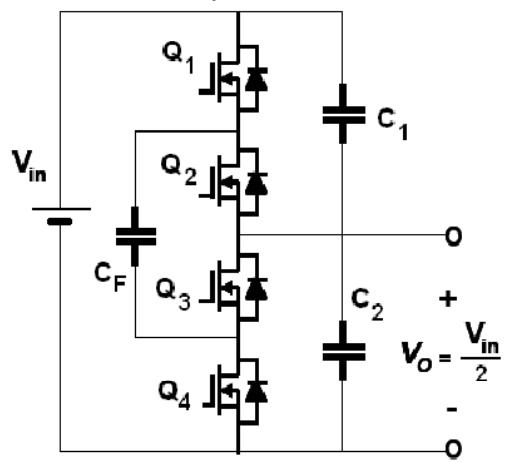

Fig. 7. Voltage divider implemented with switching capacitor converter

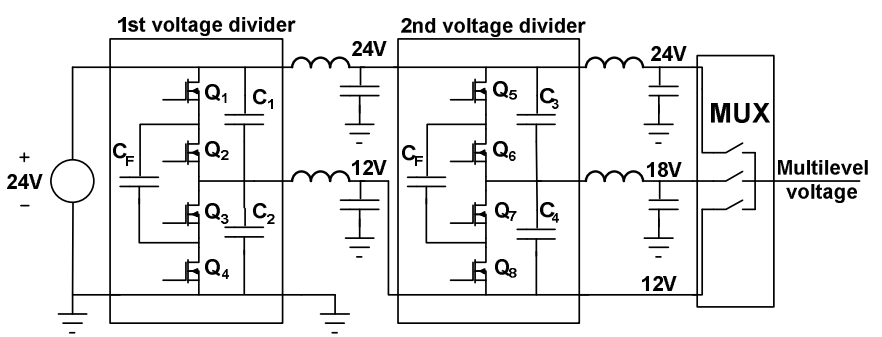

Fig. 8 Block diagram of the multilevel converter for architecture two

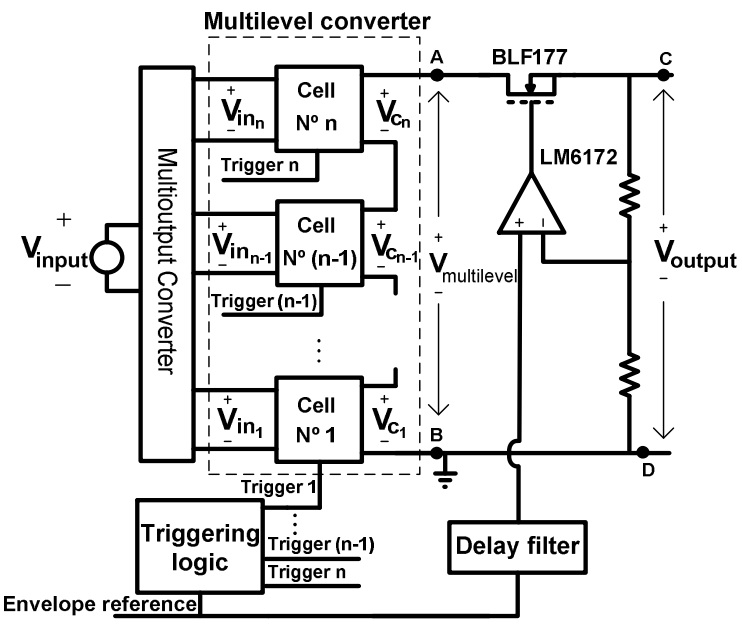

Fig. 9. Block diagram of the implemented architecture two

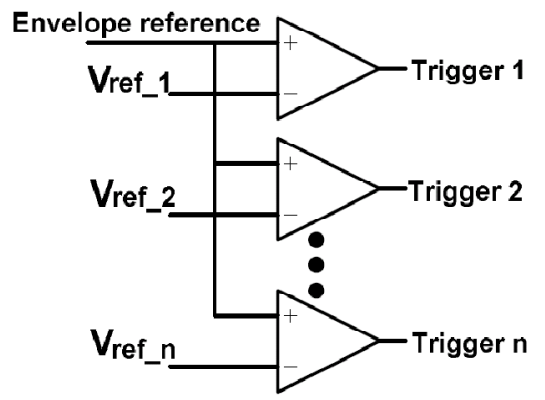

Fig. 10. Comparator logic that is used to control on/off states for each cell/switch of the multilevel converter/analog multiplexer

The reference signal that should be reproduced is sent to the analog multiplexer or the multilevel converter through the block named "triggering logic" that consists of simple comparator logic. The each voltage level is activated when the reference signal is higher than a certain value (which is different for each voltage level), Fig. 10. Consequently, the output of the multilevel converter will have discrete levels In the case of the architecture one, the number of levels will depend on the number of the used independent voltage sources and in the case of architecture two on the number of implemented cells. Each cell inside the multilevel converter and each switch inside the analog multiplexer will switch at the maximum frequency of the reference signal. Even more, the dynamic response of the multilevel converter will depend only on the speed of the diodes and MOSFETs that are used inside the switches and cells.

The same reference signal enters in the second stage and in the linear regulator (post regulator). The linear regulator reference has to be synchronized with the output voltage of the multilevel converter in order to guarantee that the system's output voltage (between points $\mathrm{C}$ and $\mathrm{D}$, Fig. 9) will be always lower than the output voltage of the multilevel converter (points A and B, Fig. 9) and, therefore, correctly reproduced. Due to the finite time to turn MOSFETs on and off, the output of the multilevel converter is delayed comparing it with the envelope reference, therefore, a delay filter which will compensate this delay is introduced between the reference signal and the linear regulator. 
In order to achieve high bandwidth of the linear regulator, it is necessary to use a high bandwidth operational amplifier in the feedback loop and to use a MOSFET with low parasitic capacitance between its gate and source as a pass element of the linear regulator.

\section{EFFICIENCY CONSIDERATIONS}

\section{First stage}

In order to obtain high overall efficiency it is necessary to maximize the efficiency of each system stage. In the case of the converters with switching capacitor the power losses are due to MOSFET switching losses and due to charge transitions through the flying capacitor. By applying the analysis like in [21], it can be shown that the losses due to the flying capacitor depend on its value and the switching frequency. By increasing the both values, the losses produced by charge transitions are reduced, but the MOSFET switching losses will increase due to higher switching frequency. Therefore, for the given value of the flying capacitor the optimal switching frequency can be found.

As a simple and robust solution for the isolated multipleoutputs dc-dc converter for the architecture two, a flyback with multiple-outputs has been selected. However, although this is very simple solution, it is not the optimal one, looking from the point of efficiency. Due to voltage fall on the secondary side diode the efficiency cannot be higher than $\mathrm{V}_{\text {cell }} /\left(\mathrm{V}_{\text {cell }}+\mathrm{V}_{\text {diode }}\right)$ (in the case when all the outputs have the same voltage). A solution that provides isolated outputs and uses synchronous rectification on the secondary side could have better efficiency. Additionally, if a single transformer is used for all the outputs its construction is another problem, especially if the high number of cells is implemented. It is necessary to optimize its design having in mind the gap and proximity effect that could increase the transformer losses. In order to minimize the losses due to the leakage inductance of the transformer, active clamping is applied.

\section{Second stage}

The losses in the second stage are due to MOSFET's and diode's conduction and switching. The conduction losses will depend only on the selection of semiconductor devices, while the switching losses will depend on the MOSFET's characteristics and supply voltage of the multilevel cell. In the case of two-level cells, the concept of multilevel converter enables the usage of low voltages in each cell, and therefore, there should be relatively low switching losses. However, the higher number of multilevel cells is, the higher conduction losses are, and consequently, there is a tradeoff, between the conduction and switching losses that must be taken into account.

\section{Third stage}

The linear regulator is supplied by the multilevel converter, and, depending on the current voltage level on its input and output, its efficiency will vary. In [18] is explained that the selection of the voltages for the multilevel converter should be done having in mind the average efficiency of the converter. Average efficiency is calculated regarding the distribution of the density of probability for the envelope of the transmitted signal. By optimizing the voltage levels in this way, the average efficiency can be improved up to $6 \%$ comparing with the equidistant voltage levels.

\section{DESIGNED SYSTEM AND EXPERIMENTAL RESULTS}

In order to compare two proposed architectures two prototypes of envelope amplifier have been made. The specifications for both prototypes are as follows:

- Variable output voltage from $0 \mathrm{~V}$ to $23 \mathrm{~V}$

- The maximum instantaneous power is $50 \mathrm{~W}$

- The maximum frequency of the reference signal is 1 $\mathrm{MHz}$

\section{A. First prototype}

The first envelope amplifier prototype consists of:

- Two converters with switching capacitor (first stage)

- Input voltage is $24 \mathrm{~V}$

- Three voltage levels are produced $(12,18$ and $24 \mathrm{~V}$ )

○ Switching frequency is $100 \mathrm{kHz}$

- Floating capacitor is $110 \mathrm{uF}$

- The maximum instantaneous power is, approximately, $50 \mathrm{~W}$

- Analog multiplexer (second stage)

- Linear regulator (post regulator).

- MOSFET BLF177 as the pass element

- Operational amplifier LM6172 for the feedback

In Fig. 11 a photograph of the prototype is presented.

Fig. 12 shows the multilevel and system's output voltage in the case of $500 \mathrm{kHz}$ and $1 \mathrm{MHz}$ sine wave. However, whenever the multilevel converter changes its output voltage there is small glitch in the output voltage. The reason is the finite bandwidth of the linear regulator. Step changes of the multilevel's voltage are composed of very high harmonics that are higher than the regulator's bandwidth. Therefore, the linear regulator is not able to react and stabilize the output voltage very well in these moments. In order to make these transitions "softer", with less high spectral components, the resistance in the gates of MOSFETs that form the analog multiplexer is increased. In this way, the MOSFET's transition time is increased, and therefore the switching loss as well, but, the linear regulator can react better and the glitch in the output voltage is almost removed.

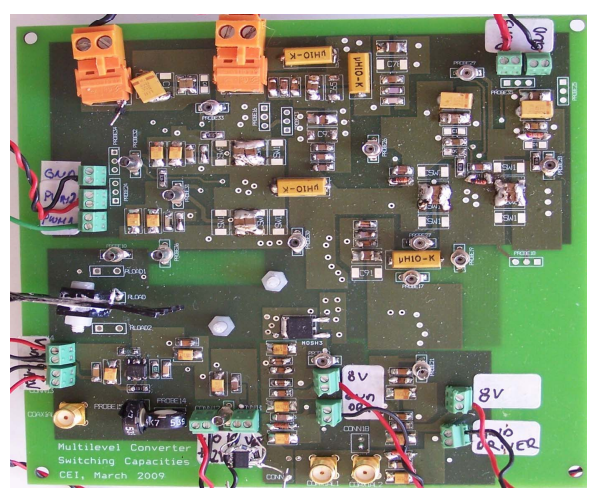

Fig. 11 Photograph of implemented multilevel converter, architecture one 


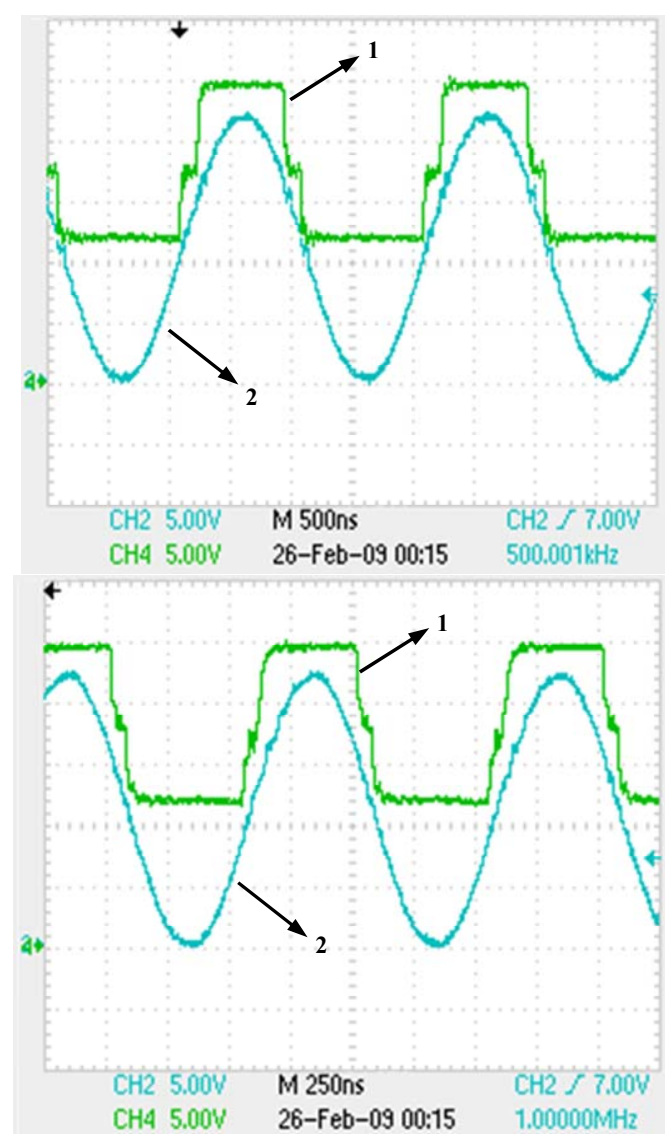

Fig. 12 Waveforms of multilevel (label 1, channel 4) and output voltage (label 2 , channel 4$)$ at $500 \mathrm{kHz}$ and $1 \mathrm{MHz}$

\section{B. Second prototype}

The second prototype's specifications are as follows:

- $\quad$ single-input multiple-outputs flyback (first stage)

- Input voltage is $24 \mathrm{~V}$

- Two $6 \mathrm{~V}$ outputs and one $12 \mathrm{~V}$ output

○ Switching frequency is $50 \mathrm{kHz}$

- The maximum instantaneous power is, approximately, $50 \mathrm{~W}$

- multilevel converter with two two-level cells (second stage)

- linear regulator (post regulator, third stage).

- MOSFET BLF177 as the pass element

○ Operational amplifier LM6172 for the feedback

In Fig. 13 pictures of the second prototype are shown.

Fig. 14 shows the multilevel and system's output voltage in the case of $500 \mathrm{kHz}$ and $1 \mathrm{MHz}$ sine wave. As in the case of the analog multiplexer, it was necessary to increase the transition time of the MOSFETs that are used in the two-level cells in order to avoid glitches in the output voltage.

\section{Efficiency measurements}

The efficiency of the system for both prototypes is measured for different sine waves and the results are summarized in Table 1. The measured efficiency is compared with theoretical efficiency of the linear regulator supplied by a constant voltage.

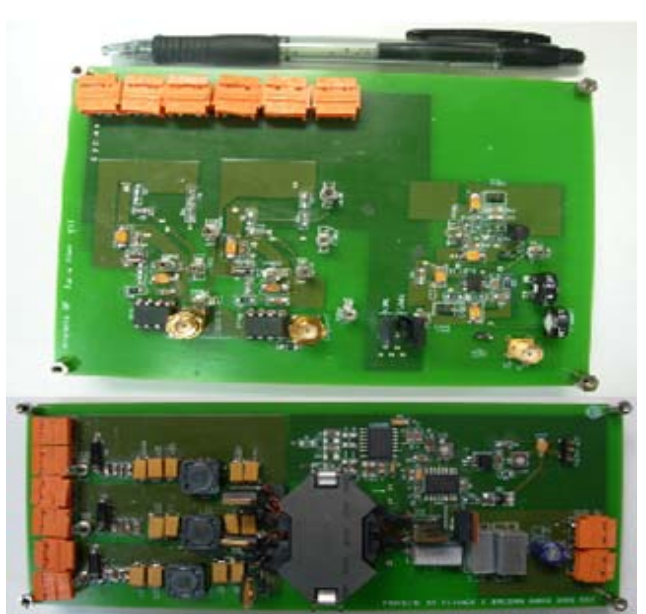

Fig. 13. Photograph of implemented multilevel converter, architecture two
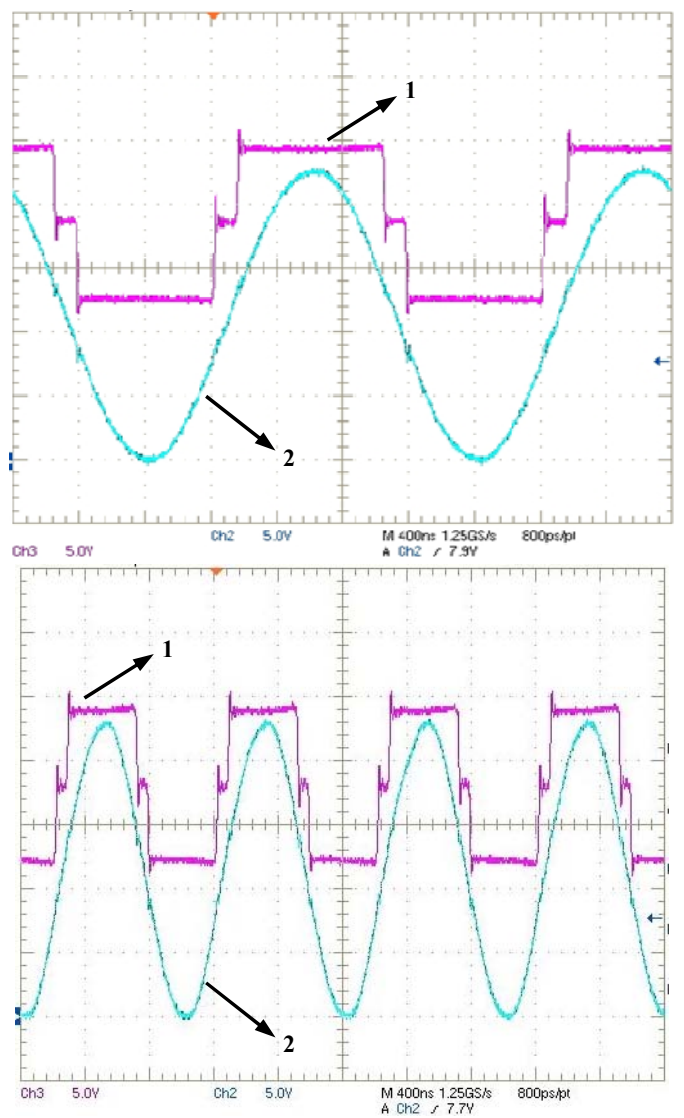

Fig. 14 Waveform of multilevel's output voltage (label 1) and linear regulator's output voltage(label 2) at $500 \mathrm{kHz}$ and $1 \mathrm{MHz}$

TABLE I

MEASURED EFFICIENCY OF THE IMPLEMENTED ENVELOPE AMPLIFIERS FOR DIFFERENT SINE WAVES COMPARED WITH THE THEORETICAL EFFICIENCY OF AN IDEAL LINEAR REGULATOR SUPPLIED BY $23 \mathrm{~V}$

\begin{tabular}{|c|c|c|c|c|}
\hline Vsin $(V)$ & $\begin{array}{c}\text { Sine wave } \\
\text { frequency } \\
(M H z)\end{array}$ & $\begin{array}{c}\text { Measured } \\
\text { efficiency of the } \\
\text { architecture one }\end{array}$ & $\begin{array}{c}\text { Measured } \\
\text { efficiency of the } \\
\text { architecture two }\end{array}$ & $\begin{array}{c}\text { Theoretical efficiency } \\
\text { of an ideal linear } \\
\text { regulator supplied by } \\
23 \mathrm{~V}\end{array}$ \\
\hline $0-9$ & 1 & $48.2 \%$ & $43.9 \%$ & $29.3 \%$ \\
\hline $5-14$ & 1 & $61.6 \%$ & $58.5 \%$ & $45.9 \%$ \\
\hline $0-22.5$ & 1 & $75.5 \%$ & $70.6 \%$ & $73.4 \%$ \\
\hline $0-9$ & 0.5 & $47.9 \%$ & $43.6 \%$ & $29.3 \%$ \\
\hline $5-14$ & 0.5 & $61.9 \%$ & $59.5 \%$ & $45.9 \%$ \\
\hline $0-22.5$ & 0.5 & $75.7 \%$ & $71.2 \%$ & $73.4 \%$ \\
\hline
\end{tabular}


TABLE II

COMPARISON OF THE IMPLEMENTED ENVELOPE AMPLIFIERS REGARDING THEIR SIZE AND WEIGHT

\begin{tabular}{|c|c|c|}
\cline { 2 - 3 } \multicolumn{1}{c|}{} & Architecture one & Architecture two \\
\hline Weight $[\mathrm{g}]$ & 215 & 420 \\
\hline Size $\left[\mathrm{cm}^{2}\right]$ & 217.5 & 297 \\
\hline
\end{tabular}

Both multilevel solutions have better efficiency than linear regulator when signals with small average value are transmitted, and that is mostly the case when the EER technique is applied. The efficiency of the envelope amplifier is constant (around $43 \%$ and $48 \%$, depending on the implementation) when small signals are reproduced, the reason is that only the $12 \mathrm{~V}$ cell is active, and there is not any switching losses, only conduction losses, regardless on the frequency of the sine wave. Additionally, the efficiency of the envelope amplifier implemented with switching capacitor is significantly higher than the efficiency of the envelope amplifier that is made by employing a flyback converter.

In Table 2 a comparison regarding the size and weight of the realized envelope amplifier is made.

\section{CONCLUSIONS}

In this paper two solutions for power supply for EER technique are compared. Both solutions are composed of a multilevel converter that is put in series with a linear regulator. First solution is based on the multilevel converter composed of two switching capacitor converter, and the second solution is based on single-input multiple-output flyback converter. Both prototypes can deliver up to $50 \mathrm{~W}$ of instantaneous power and reproduce sine wave up to $1 \mathrm{MHz}$. The system's efficiency for both solutions has been measured for the various $1 \mathrm{MHz}$ and $0.5 \mathrm{kHz}$ sine waves and compared with the efficiency of the ideal linear regulator. When the sine wave has small average value (what is usually the case in the case of RF amplifier) both envelope amplifiers have better efficiency up to $19 \%$ than linear regulator. It is shown that the architecture based on switching capacitor converters has better efficiency up to $5 \%$ and it is smaller and lighter. Additionally, this architecture is lighter, smaller and does not need any big inductive component comparing with flyback converter and it can be integrated easily.

\section{REFERENCES}

[1] S.Roy, "Energy Logic for Telecommunications", white paper for Emerson Network Power, September 2008

[2] G.Pierre, "Power System Efficiency in Wireless Communication", Applied Power Electronics Conference, APEC '06, special presentations

[3] F.H. Raab, D.J. Rupp, "High efficiency single sideband HF/VHF transmitter based upon envelope elimination and restoration", Sixth Intern. Conference on HF Radio Systems and Techniques, 4-7 July, 1994, Pages:21-25

[4] F.H. Raab, B.E.Sigmon, R.G.Myers, R.M. Jackson, "L-Band Transmitter Using Kahn EER Technique", Transactions on Microwave Theories and Techniques, Volume 46, Issue 12, Part 2, Pages: 2220-2225, December 1998.

[5] F.H.Raab, P.Asbeck, S.Cripps, P.B.Kenington ,Z.B.Popovic, N.Pothecary, J.F.Sevic, N.O.Sokal ,"RF and Microwave Power Amplifier and Transmitter Technologies - Part 1", High Frequency Electronics, Vol. 2, No.3, Pages:22-36, May 2003

[6] F.H. Raab, P. Asbeck, S. Cripps, P.B. Kenington, Z.B. Popovic, N. Pothecary, J.F. Sevic, N.O. Sokal, "Power Amplifiers and Transmitters for RF and Microwave"
[7] A.A.M. Saleh, D.C. Cox, "Improving the Power Added Efficiency of FET Amplifiers Operating with Variying-Envelope Signals",Trans. on Microwave Theories and Techniques, vol. MTT-31, no.1, pp.51-56, Jan. 1983.

[8] F.H. Raab, "Efficiency of Envelope-tracking RF Power Amplifier Systems", Proc.. RF Expo East'86, pp. 303-311.

[9] P. Midya, K. Haddad, L. Connell, S. Bergstedt, B. Roeckner, "Tracking power converter for supply modulation of RF power amplifiers," IEEE Power Electronics Specialists Conference, PESC. 2001, Vol. 3, Pages: $1540-1545$

[10] J. Staudinger, B. Gilsdorf, D. Newman, G. Norris, G. Sadowniczak, R. Sherman, T. Quach, "High efficiency CDMA RF power amplifier using dynamic envelope tracking technique," Microwave Symposium Digest., IEEE MTT-S International, Vol. 2, June 2000, Pages: 873-876

[11] A. Soto, J.A. Oliver, J.A. Cobos, J. Cezon, F. Arevalo, "Power supply for a radio transmitter with modulated supply voltage", Applied Power Electronics Conference, APEC '04, Volume: 1, Feb. 2004 Pages:392 398

[12] V. Yousefzadeh, E. Alarcon, D. Maksimovic, "Three-level buck converter for envelope tracking in RF power amplifiers," IEEE Trans. on Power Electronics, Volume:21, Issue: 2, March 2006, Pages:549- 552

[13] US Patent No. 6084468, Method and Apparatus for High Efficiency Wideband Power Amplification, July 2000.

[14] V. Yousefzadeh, E. Alarcon, D. Maksimovic, "Efficiency optimization in linear assisted switching power converters for envelope tracking in RF power amplifiers", IEEE International Symposium on Circuits and Systems, ISCAS 2005, 23-26 May, pages:1302-1305 Vol. 2

[15] V. Pinon, F. Hasbani, A. Giry, D. Pache, C. Gamier, "A Single-Chip WCDMA Envelope Reconstruction LDMOS PA with $130 \mathrm{MHz}$ SwitchedMode Power Supply," Solid-State Circuits Conference, 2008. ISSCC 2008. Digest of Technical Papers. IEEE International, vol., no., pp.564636, 3-7 Feb. 2008

[16] P.G. Blanken, R. Karadi, H.J. Bergveld, "A 50MHz Bandwidth MultiMode PA Supply Modulator for GSM, EDGE and UMTS Application", IEEE Radio Frequency Integrated Circuits Symposium, RFIC 2008, 15-17 June 2008

[17] L.Marco, E. Alarcon, D. Maksimovic, "Effects of switching power converter nonidealities in Envelope Elimination and Restoration technique", IEEE International Symposium on Circuits and Systems, ISCAS 2006, 21-24 May 2006

[18] M.Vasic, O.Garcia, J.A.Oliver, P.Alou, D.Diaz, J.A.Cobos, "Multileve Power Supply for High Efficiency RF Amplifier", Proc. of the 24th Annual IEEE Applied Power Electronics Conference, APEC '09, February 2009

[19] Gong, G., Ertl, H., Kolar, J. W., "A Multi-Cell Cascaded Power Amplifier", Proceedings of the 21st Annual IEEE Applied Power Electronics Conference and Exposition (APEC'06), Dallas (Texas), USA, March 19 - 23, Vol. 3, pp. 1550 - 1556

[20] J.Sun, M.Xu, Y.Ying, F.C.Lee, "High Power Density, High, Efficiency System Two-stagePower Architecture for Laptop Computers", 37th IEEE Power Electronics Specialists Conference / June 18 - 22, 2006, Jeju, Korea

[21] S. Ben-Yaakov, "Switched Capacitors Converters", Professional Education Seminar at the 24th Annual IEEE Applied Power Electronics Conference, APEC '09, February 2009 